Cuadernos de $\mathrm{H}$ ideas

ISSN: 2313-9048

cuadernosdehideas@perio.unlp.edu.ar

Universidad Nacional de La Plata

Argentina

\title{
La Asignación Universal por Hijo y las representaciones de las madres perceptoras. Estudio de casos en Villa Argüello (Berisso)
}

Morzilli, Melina

La Asignación Universal por Hijo y las representaciones de las madres perceptoras. Estudio de casos en Villa Argüello (Berisso)

Cuadernos de $\mathrm{H}$ ideas, vol. 13, núm. 13, e020, 2019

Universidad Nacional de La Plata, Argentina

DOI: https://doi.org/10.24215/23139048e020

Esta obra está bajo una Licencia Creative Commons Atribución-NoComercial-Compartirlgual 4.0 Internacional. 


\title{
Artículos
}

\section{La Asignación Universal por Hijo y las representaciones de las madres perceptoras. Estudio de casos en Villa Argüello (Berisso)}

The Universal Assignment by Son and the representations of the mothers of children perceivers. The case of Villa Argüello

(Berisso)

\author{
Melina Morzilli melinamorzilli@gmail.com \\ UNAJ-CONICET/IDIHCS-UNLP, Argentina
}

Resumen: El siguiente trabajo tiene como objetivo dar cuenta de la incidencia de la Asignación Universal por Hijo (AUH), en las representaciones de las madres perceptoras de la misma con hijos/as que asisten a una Escuela Primaria ubicada en el barrio Villa Argüello (Berisso, Provincia de Bs. As.). Para ello, se propuso comprender las representaciones de dichas madres respecto de la escolaridad en el nivel primario de sus hijos/as y, de las políticas sociales en general y de la AUH en particular, observando los cambios y/o continuidades en las respectivas representaciones a partir de la percepción de la AUH. El trabajo se realizó mediante un enfoque metodológico cualitativo basado en entrevistas en profundidad y observación etnográfica como técnicas principales de recolección/construcción de datos. Se concluyó que a partir de la percepción de la $\mathrm{AUH}$, hubo tanto cambios como continuidades en las representaciones de las madres. En ambos casos, se observó una incidencia positiva por parte de la AUH en las representaciones escolares y en las concepciones sobre las políticas sociales.

Palabras clave: AUH, Berisso, Madres, Representaciones .

Abstract: The following work aims to account for the incidence of Universal Assignment by Child (AUH), in the representations of the mothers who receive it with children who attend a Primary School located in the Villa Argüello neighborhood (Berisso, Province of Bs. As.). To do this, it was proposed to understand the representations of these mothers with respect to schooling at the primary level of their children, and of social policies in general and the AUH in particular, observing the changes and / or continuities in the respective representations from the perception of the AUH. The work was carried out through a qualitative methodological approach based on in-depth interviews and ethnographic observation as main data collection / construction techniques. It was concluded that from the perception of the AUH, there were both changes and continuities in the representations of the mothers. In both cases, a positive incidence was observed by the AUH in the school representations and in the conceptions about the social policies.

Keywords: AUH, Berisso, Mothers, Representations .

\section{Introducción}

En el presente trabajo se presentan los resultados provenientes del estudio de la incidencia de la Asignación Universal por Hijo (AUH), en las representaciones de las madres perceptoras de la misma con hijos/as que asisten a una Escuela Primaria ubicada en el barrio Villa Argüello (Berisso, 
Provincia de Bs. As.), con respecto a la escolaridad en el nivel primario de sus hijos/as y respecto a las políticas sociales en general y a la AUH en particular. El objetivo del trabajo radica en dar cuenta de, en primer lugar, las características de la institución educativa a la que asisten; en segundo lugar, las características socio-ocupacionales y educativas de los hogares de los/as sujetos estudiados/as; en tercer lugar, los significados que las madres le otorgan a la escolaridad de sus hijos/as durante los estudios primarios; en cuarto lugar, los significados que las madres le otorgan a las políticas sociales en general a y a la AUH en particular; en quinto lugar, las representaciones del personal directivo y docente de la Escuela a la cual asisten estos niños/as con respecto a la incidencia de la AUH en las representaciones escolares de las madres seleccionadas. Por último, se propuso observar los cambios y/o continuidades en las representaciones con respecto a la escolaridad y a las políticas sociales a partir de la percepción de la AUH. Para ello, el trabajo se realizó mediante un enfoque metodológico cualitativo basado en entrevistas en profundidad (semiestructuradas) y observación etnográfica como técnicas principales de recolección/construcción de datos.

Es necesario señalar que el contexto previo a la creación de la AUH se remonta a la crisis económica y política que se vivió en Argentina en 2001. Dicha crisis fue consecuencia del agonizante modelo neoliberal (1) que provocó elevados índices de desocupación, pobreza e indigencia, los cuales a su vez fueron profundizados por la crisis de la convertibilidad y su salida devaluatoria. En este contexto los sectores en situación de vulnerabilidad social, (2) reclamaron al Estado la implementación de planes sociales de alcance masivo que posibilitaran ingresos mínimos de subsistencia (Rapoport, 2010; Féliz, 2012 a; b). Siendo, entonces, la conflictividad de los años de crisis la que forzó la implementación del Plan Jefes y Jefas de Hogar Desocupados en 2002, este plan tuvo un carácter masivo (incluyó a 2 millones de beneficiarios) y otorgó un ingreso de $\$ 150$ con contraprestaciones mínimas. La transformación del Plan Jefes en planes más segmentados, como el Plan Familias que mantenía los mismos niveles de gasto social pero para un menor número de beneficiarios, provocó nuevas reacciones de parte de los sectores vulnerables que, ya durante el gobierno kirchnerista tuvieron respuesta estatal mediante el Programa Argentina Trabaja y la Asignación Universal por Hijo (Gasparini \& Cruces, 2010; Féliz, 2012 a; b).

Cabe recordar, que la $\mathrm{AUH}$ es una política que se caracteriza por combinar por un lado, las condicionalidades de los tradicionales programas de transferencias condicionadas de ingresos (PTCI) ) (3) precedentes en términos de salud y educación y, por otro, la lógica de seguros sociales de la protección social en general -por su carácter de asignación familiar- y finalmente, un marco de ampliación de derechos de los trabajadores en general y de los/as niños/as y jóvenes en particular (D’Amico, 2013).

Es a partir de noviembre de 2009 que se crea la AUH por decreto del Poder Ejecutivo Nacional, la cual comienza a ser percibida por madres, padres y/o tutores de menores de 0 a 18 años y/o con hijos 
con discapacidad sin límites de edad, que se encuentren en situación de informalidad laboral, desempleo o formalidad pero que su salario sea inferior al salario mínimo vital y móvil. Dicha política consiste en un pago mensual de dinero cuyo titular de derecho son los hijos menores de 18 años y chicos discapacitados sin límite de edad. Con la misma, el Estado, busca asegurarse de que los niños y adolescentes asistan a la escuela, realicen controles periódicos de salud y cumplan con el calendario de vacunación obligatorio, ya que éstos son requisitos condicionales para cobrarla. Lo perceptores tendrán depositados el $80 \%$ del valor de la Asignación correspondiente por cada hijo. Para tener derecho a la percepción del 20\% acumulado del año anterior, deberá acreditarse el cumplimiento de los controles sanitarios, del Plan de Vacunación Obligatorio y la inscripción al Plan Nacer / Programa SUMAR; y además deberá acreditarse la concurrencia a establecimientos educativos públicos (Decreto 1602/09) (4)

Con respecto a las condicionalidades, los fundamentos de las mismas se apoyan, entre otras, en las siguientes premisas:

“...apostar al desarrollo del capital humano y social... la exigencia de condicionalidades asegura una responsabilización de las familias respecto de la educación y salud de sus integrantes... apuesta a la cultura del esfuerzo familiar en la medida en que está vinculada al cumplimiento de condicionalidades familiares y del niño estableciendo un equilibrio entre derechos (el beneficio) y obligaciones (las contraprestaciones)... la educación es un factor estratégico para abatir la pobreza y la desigualdad, apoya el desarrollo de credenciales suficientes para los jóvenes a fin de obtener ingresos que puedan mejorar la calidad de vida de sus familias y revitalización del rol de la mujer como factor decisivo en el desarrollo familiar y comunitario..." (Novacovsky y Sobron, 1999).

Es decir que, las condicionalidades tienen el objeto de aumentar la acumulación del capital humano y consecuentemente romper el círculo intergeneracional de la pobreza; al mismo tiempo buscan reforzar el vínculo entre derechos y obligaciones (Straschnoy, 2015). Por esta razón es que interesa aquí analizar la incidencia de la AUH en las representaciones escolares de madres con hijos/as que se encuentren en situación de vulnerabilidad social y económica.

A su vez, se ha propuesto analizar las representaciones de las madres perceptoras de la $\mathrm{AUH}$, debido a que son ellas en general las encargadas del cuidado de sus hijos/as. Además, la AUH privilegia la percepción por parte de las madres antes que la de su padre u otro tutor (Polischer, 2012).

Se ha seleccionado el barrio de Villa Argüello (Berisso, Prov. Bs. As.) debido a que en el mismo existe un asentamiento con viviendas precarias y personas que viven en situación de vulnerabilidad social y económica. Este asentamiento que abarca en la actualidad unas 50 cuadras, nació con el barrio, sin embargo fue aumentando su tamaño durante las décadas de gobiernos neoliberales. Puntualmente dicho asentamiento es la residencia de los hogares de madres objeto de análisis.

Por último, se ha elegido una Escuela Primaria en particular, porque es la institución educativa de nivel primario más cercana al asentamiento.

Ahora bien, al analizar las investigaciones existentes sobre la $\mathrm{AUH}$ y su incidencia en la escolaridad, se ha observado que la mayoría de 
los estudios existentes hasta el presente abordaron principalmente la incidencia de la AUH sobre diferentes dimensiones de la escolaridad, empero sin embargo no se han problematizado las representaciones sobre la educación primaria, como tampoco sobre las políticas sociales en general y sobre la AUH en particular, por parte de las madres perceptoras de la misma, ni las representaciones del personal directivo de las instituciones educativas del nivel primario con respecto a la incidencia de la AUH en las representaciones escolares de estas madres perceptoras desde el paradigma constructivista.

Por lo tanto, sería enriquecedor abordar estudios que ayuden a pensar herramientas de inclusión socioeducativa que tomen como sujetos de análisis a poblaciones que se encuentran subordinadas por su posición social (Terigi, 2009).

A continuación, se señalan los antecedentes y el marco teórico; luego se expone la metodología y las fuentes; seguidamente, se describen las características de la institución educativa a la que asisten los hijos/ as de las madres seleccionadas y Caritas; luego, las características socio- ocupacionales y educativas de los hogares de dichas madres; posteriormente se presentan los significados que las madres le otorgan a, la escolaridad de sus hijos/as durante los estudios primarios, a la $\mathrm{AUH}$ y a las políticas sociales en general; luego se analizan las representaciones del personal directivo y docente de la Escuela a la cual asisten los hijos/as de estas madres con respecto a la incidencia de la AUH en las representaciones y finalmente, se realizan las reflexiones finales observando la incidencia de la AUH en las respectivas representaciones y por último, los cambios y/o continuidades a partir de la misma.

\section{Antecedentes y marco teórico}

Se ha encontrado en la revisión bibliográfica que la dimensión educativa de la $\mathrm{AUH}$ ha sido más profusamente estudiada en relación a otras dimensiones. Dentro del abordaje metodológico cualitativo, algunas investigaciones (Pereyra y Pereyra, 2010) se propusieron conocer la incidencia que tuvo la AUH en la educación y en la salud de los niños de las familias pertenecientes a los barrios Campo Contreras y 8 de Abril, de la ciudad de Santiago del Estero. Con respecto a la educación, concluyeron que hubo un incremento de los niños matriculados en los establecimientos educativos durante el año 2010 en relación a los años anteriores. Otras (Ministerio de Educación Presidencia de la Nación, 2011), analizaron las estrategias educativas desarrolladas en los distintos niveles para la puesta en marcha de la AUH y las acciones desarrolladas en el ámbito educativo por los múltiples sujetos sociales involucrados. Observaron, que la AUH ha logrado incorporar al sistema educativo a un conjunto de la población que no concurría a la escuela, y, a su vez, ha mejorado sustancialmente las condiciones de esa asistencia, contribuyendo a generar "conciencia" respecto de la importancia de la asistencia escolar de modo más potente que las leyes educativas mismas. Mientras que, Glutz y Moyano (2014), analizaron el proceso 
de implementación de la AUH y los primeros impactos respecto a la educación en el nivel central de la provincia de Buenos Aires y en dos distritos: José C. Paz y General Pueyrredón. Sostuvieron que la asignación fue una herramienta que facilitó el cumplimiento de la obligatoriedad escolar al comprometer a los padres a enviar a los hijos a la escuela para cobrar la asignación. Por su parte, Mouriño (2013) avanzó sobre el estudio del derecho a la educación en la Primera Infancia y la AUH en el sur de la Ciudad de Buenos Aires (Villa Soldati). La hipótesis de su trabajo giró en torno a la tensión que se presenta entre un sistema que proclama cobertura universal, y la escasa oferta de educación pública destinada para la primera infancia en la Ciudad de Buenos Aires.

Siguiendo el abordaje metodológico cualitativo, Alonso y Santiago (2014) realizaron reflexiones sobre la asignación universal por hijo desde la escuela en el nivel secundario en la ciudad de Córdoba. Según las autoras la AUH funcionó como dispositivo de re-conocimiento e inclusión por parte del estado, basado en la reactivación social y económica que generó expectativas en el futuro laboral de los jóvenes. Mientras que otras investigaciones (Gluz et al. 2014a; 2014b), se focalizaron en el nivel inicial avanzando sobre los sentidos de la inclusión escolar que se configuraron a partir del diseño y apropiación de la política $\mathrm{AUH}$, en la Provincia de Buenos Aires, y señalaron al igual que Mouriño (2013) los límites de la oferta educativa en el nivel inicial a través de la falta de vacantes en algunos distritos.

Por último, Montes y otros (2014) analizaron la incidencia de la AUH en las instituciones escolares de Mendoza (nivel inicial, primario y medio). Sus conclusiones fueron que a partir de la subjetividad de los agentes involucrados en la ejecución de la $\mathrm{AUH}$ respecto de la representación simbólica de la asignación, se evidenciaron diferencias en los informantes. Argumentos conservadores: "Para mi no ha sido beneficiosa para los chicos porque los papás usan a sus hijos como un instrumento; como un modo de poder cobrar una asignación” (preceptora escuela media). Argumentos de corte más comunitario: "Es un derecho y una herramienta con la que puedo acompañar a esta familia para que el chico esté escolarizado" (director de CEOS).

En lo que respecta a las investigaciones que realizaron un abordaje metodológico cuantitativo, se ha encontrado un estudio (Subsecretaría de Planeamiento Educativo de la Nación, 2010) que relevó 676 escuelas (el 69\% pertenecían a la región del Centro, el 17\% al Sur, el $8 \%$ a la región NEA, el $4 \%$ Cuyo y el $2 \%$ al NOA), y concluyó que el $51 \%$ de los directivos encuestados afirmaron que se elevó la matrícula escolar. $\mathrm{Al}$ igual que otros estudios (Pereyra y Pereyra, 2010), aunque desde otro método sostuvieron que la matrícula inicial aumentó un $15 \%$, la primaria un 10\%, y la del nivel secundario aumentó un 19\%. Por otro lado, y en contraste con las anteriores investigaciones, Tuñón y González (2011) señalaron la ineficacia de la AUH en cuanto a la escolarización secundaria de los adolescentes en los grandes aglomerados urbanos de Argentina. Discrepando de este modo con otras investigaciones (Alonso y Santiago, 2014). Más adelante, Salvia y otros (2013), realizaron un 
análisis de la incidencia de la AUH en materia de inseguridad alimentaria y déficit educativo en Argentina. En el caso específico de la educación primaria, advirtieron una leve caída de inasistencia escolar de niños/as de 6 a 12 años habría sido independiente de los incentivos generados por los ingresos del régimen de AUH. Otro estudio (Campanella, et al., 2015), sobre la incidencia en los niveles de inclusión social de dos políticas públicas en el área educativa (Ley De Educación Nacional y la Asignación Universal por Hijo para Protección Social), observó los movimientos en la matrícula escolar de todos los niveles en Argentina, y encontró que existió relación entre la implementación de políticas públicas en esta área, la asignación de recursos del presupuesto educativo y la cantidad de estudiantes matriculados.

Por su parte, desde una metodología mixta, Vega y otros (2011) evaluaron la incidencia de la AUH, su forma de implementación y contribución al desarrollo integral, salud, educación. Su muestra fue la ciudad de Mar del Plata. Concluyeron que la matrícula del nivel secundario se incrementó en un $25 \%$, aunque en contraste a lo que sostuvo el estudio del Ministerio de Educación Presidencia de la Nación (2011), afirmaron que no se conocen aún estadísticas de permanencia y calidad educativa, concordando en este sentido con Pereyra y Pereyra (2010).

Por otra parte, Arroyo (2014), se propuso analizar la AUH desde la perspectiva de las familias titulares de derecho, buscando indagar las transformaciones en la economía doméstica, la escolaridad y la salud de las mismas incorporando la voz de las mismas. Analizó familias de la ciudad de Mar del Plata (partido de General Pueyrredón). Concluyó que según la experiencia de los entrevistados "las condicionalidades son valoradas positivamente por la mayoría de los entrevistados y son asociadas a un mejoramiento de las condiciones de salud y educación en los respectivos niños perceptores de la AUH".

En consideración a las investigaciones enunciadas, se ha observado que los autores avanzaron sobre el tema referido a la escolaridad, empero, los estudios que avanzaron en el análisis de la AUH desde una metodología cuantitativa, mostraron parcialmente un fenómeno social que presenta una complejidad mayor obviando así la visión de los propios sujetos destinatarios de la AUH. En cuanto a aquellos que investigaron desde lo cualitativo, se centraron en la educación y la salud, o bien solamente en la educación como derecho, o en la matrícula escolar adolescente urbana, o en el nivel inicial de la educación, y aquellos que abarcaron los tres niveles, lo hicieron sólo desde la subjetividad de los agentes involucrados en la ejecución de la AUH. En síntesis, si bien los autores concluyeron que hubo un aumento en la matrícula escolar luego de la implementación de la AUH, llama la atención que en los citados estudios no se ha investigado las representaciones de las madres perceptoras en cuanto a los significados de la AUH y de la escolaridad de sus hijos/as, ni del personal de las instituciones escolares a las que concurren, como tampoco se ha problematizado la asistencia escolar; es decir, no se han preguntado desde el paradigma teórico constructivista (Valles, 1997) si los/as alumnos/as concurren sostenidamente a la escuela (o si por el contrario, abandonan), 
soslayando de este modo la visión de los/as agentes, que es fundamental para generar un conocimiento amplio sobre el grado de incidencia de la AUH en tales cuestiones.

El presente trabajo parte del paradigma constructivista, el cual surge criticando al objetivismo, al realismo empírico y al esencialismo. Dicho paradigma sostiene que la realidad y la verdad, no sólo se descubren, sino que se construyen; es decir, son "producto de prácticas discursivas complicadas" (Valles, 1997).

En cuanto al marco teórico que oriental mismo, está centrado en la propuesta teórica de Bourdieu denominada constructivismo estructuralista, con la cual se busca superar la falsa dicotomía objetivismo/ subjetivismo. Por estructuralismo o estructuralista, el autor entiende que existen en el mundo social mismo, y no solamente en los sistemas simbólicos, el lenguaje, mito, etc., estructuras objetivas, independientes de la conciencia y la voluntad de los agentes, que son capaces de orientar o de coaccionar sus prácticas o sus representaciones. Mientras que por constructivismo, quiere decir que hay una génesis social de una parte de los esquemas de percepción, de pensamiento y de acción que son constitutivos de lo que llamamos habitus, y por otra parte estructuras, y en particular lo que llama campos y grupos, especialmente de lo que se llama generalmente las clases sociales (Bourdieu, 1988).

Con respecto a la dimensión de análisis de las representaciones y resignificaciones de las madres, como del personal docente, se propone el concepto de representación social definido por Jodelet (1986). Para la autora las representaciones sociales corresponden a una forma específica de conocimiento, el conocimiento ordinario, que es incluido en la categoría del sentido común y tiene como particularidad la de ser socialmente construido y compartido en el seno de diferentes grupos. Esta forma de conocimiento tiene una raíz y un objetivo práctico: apoyándose en la experiencia de las personas, sirve de grilla de lectura de la realidad y de guía de acción en la vida práctica y cotidiana. Son los significados y las prácticas. La representación incide en el comportamiento social; es un punto donde se intersectan lo psicológico y lo social, y constituye el saber de sentido común; son modalidades de pensamientos prácticos orientados hacia la comunicación, comprensión y dominio del entorno social, material e ideal. En efecto, ya sea un conocimiento intersubjetivo o situado en el espacio público, va a contribuir a producir y mantener una visión común a un grupo social, ya se trate de una clase social, de un grupo cultural (Jodelet, 1986).

\section{Metodología y fuentes}

Para poder dar cuenta de la incidencia de la AUH en las representaciones de las madres seleccionadas, con respecto a la escolaridad del nivel primario de sus hijos/as como a su vez, en las representaciones sobre las políticas sociales en general y en la AUH en singular; para así, dar cuenta de la incidencia de la AUH en las mismas y de los cambios y/o continuidades en dichas dimensiones, se propone abordar la investigación 
partiendo de la triple adscripción social que adquieren las agentes aquí estudiadas: madres (5) perceptoras de la $\mathrm{AUH}$, cuidadoras e integrantes de familias en situación de vulnerabilidad social y económica, y madres de alumnos/as de una Escuela Primaria ubicada en Villa Argüello (Berisso, Prov. De Bs. As.).

Debido a que la autora del presente trabajo, conoce en profundidad el barrio Villa Argüello por lazos sociales previamente establecidos a la presente investigación, con diferentes actores sociales del mismo (directivos de la única Escuela Primaria del barrio; personal de caritas que trabaja en la parroquia colindante a la Escuela y, diferentes vecinos que nacieron en el barrio hace más de 50 años), se tomó contacto con la Institución escolar denominada Escuela Primaria Nº "Martín Miguel de Güemes" ubicada en el respectivo barrio, ya que la misma recibe un alto porcentaje de niños/as en situación de vulnerabilidad social y económica, los/as cuales perciben la $\mathrm{AUH}$ y, en donde la mayoría de ellos/as viven en un asentamiento ubicado en las cercanías de dicha Escuela. A su vez, también se ha tomado contacto con personal de Caritas que trabaja en la Parroquia San Miguel Arcángel, ubicada en zona colindante a la Escuela, puesto que las madres seleccionadas se acercaban a dicho lugar todos las semanas para obtener ropa, zapatos y diferentes alimentos para su familia.

Por la naturaleza de los problemas que interesaba indagar, se realizó un estudio cuya estrategia metodológica fue de carácter cualitativa, con el fin de captar las diferentes dimensiones co-construidas con respecto a la significación de los aspectos que nos interesan estudiar. Es característico de los estudios cualitativos escoger pocos casos para poder profundizar en su observación y análisis. La riqueza de este estudio no pretendió fundamentarse en su representatividad entendida en términos estadísticos, sino en su significatividad en términos de la capacidad de la aproximación que se realice, para mostrar la articulación de elementos que configuran la singularidad del problema en cada caso. Para ello, se eligió una muestra teórica intencionada por bola de nieve conformada por 20 madres cuyos hijos/as asisten a la Escuela Primaria ubicada en el barrio Villa Argüello y, se utilizó como técnicas de recolección/ construcción de datos entrevistas en profundidad /semi-estructuradas) y observación etnográfica durante el período 2013-2014.(6) A su vez, se entrevistaron en la Escuela a 1 directivo, 2 docentes y 1 portero. A través de la utilización de estas técnicas tanto en la enunciada Escuela y como en Caritas de la Parroquia San Miguel Arcángel se buscó construir la siguiente información: las características de la institución educativa a la que asisten; las características socio-ocupacionales y educativas de los hogares de las sujetos estudiadas; los significados que las madres le otorgan a la escolaridad de sus hijos durante los estudios primarios; los significados que las madres le otorgan a las políticas sociales en general y a la $\mathrm{AUH}$ en particular; las representaciones del personal directivo y docente de la Escuela a la cual asisten los hijos/as de dichas madres con respecto a la incidencia de la $\mathrm{AUH}$ en las representaciones de las madres seleccionadas. 


\section{El Barrio, la Escuela, Caritas y los Hogares}

Como se ha indicado anteriormente, este trabajo parte del marco teórico propuesto por Bourdieu denominado estructuralismo-constructivista, por lo cual se hace necesario describir el contexto estructural de los agentes analizados. Es decir, aquí se describirán el barrio, la escuela, la parroquia y los hogares de las madres seleccionadas.

\section{Acerca del Barrio}

Villa Argüello (VA) es un barrio cuyo origen se remonta a la década del 40 'del siglo XX. El mismo está ubicado en el Partido de Berisso (Provincia de Buenos Aires), comprendido entre las calles 60, 122, el camino Juan Domingo Perón y calle 135 (Figura 1). Los datos censales de 2001 indican 5.662 habitantes en VA (Deambrosi, 2009). Desde la fundación del barrio, se establecieron en una parte del barrio entre las calles 64 y 126 hasta 66 y Montevideo, un asentamiento con viviendas precarias, el cual se fue ampliando durante las décadas de gobiernos neoliberales. En sus inicios se lo denominaba Barrio Chino (7). Los hogares en situación de vulnerabilidad social y económica que viven allí, atravesaron momentos en los que la política social de gobiernos de la década de los 90 era poco significativa, y luego fueron testigos y beneficiarios de políticas sociales que se comenzaron a implementar a partir del Neodesarrollismo (8) (Féliz, 2012 a;b; Rapoport, 2012). A su vez, como se ha señalado con anterioridad, se eligió dicho barrio ya que por un lado, la autora tenía acceso a los sujetos sociales seleccionados, y por otro lado, debido a que el mismo no fue incorporado en el informe de las cinco Universidades Nacionales (Ministerio de Educación Presidencia de la Nación, 2011), a pesar de ser un barrio en el cual la única Escuela Primaria que tiene posee una matrícula elevada de alumnos en situación de vulnerabilidad social y económica.

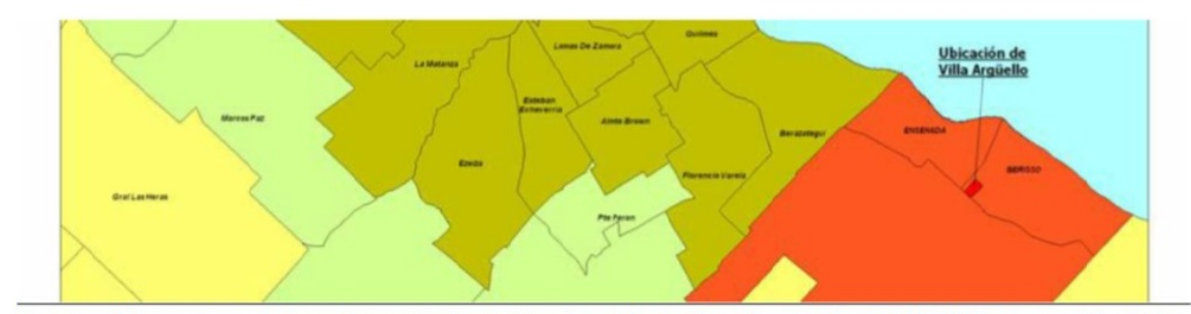

Fuente: elaboración propia en base a información obtenida de Deambrosi, N. (2009) 'Ni Berisso, ni La Plata: Villa Argüello': Territorio y organización social en un barrio del Gran La Plata 1958- 2008 [en línea]. Trabajo final de grado. Universidad Nacional de La Plata. Facultad de Humanidades y Ciencias de la Educación. Disponible en: http://www.memoria.fahce.unlp.edu.ar/tesis/te.575// te.575.pdf

\section{Figura 1}

Ubicación del Barrio Villa Argüello, Berisso, Provincia de Buenos Aires. 


\section{Acerca de la Escuela y Caritas}

Para obtener respuesta al problema que se ha observado y poder dar cuenta de la incidencia de la AUH en las representaciones escolares del nivel primario de las madres como a su vez, las percepciones que tiene el personal de la Escuela sobre la respectiva temática, primero, se concurrió a la Escuela Primaria $N^{\circ} 8$ General Martín Miguel de Güemes fundada en 1938, la cual está ubicada en el barrio de Villa Argüello en la calle 63 esquina 125 de la localidad de Berisso, ya que a dicha institución asisten los/as hijos/as de las madres objeto de nuestro estudio, y realizamos allí entrevistas a los directivos, asistentes sociales, docentes y personal auxiliar.

Según la información que brindó el personal directivo de la escuela, la misma tenía una matrícula escolar de alrededor de 400 alumnos, con una tasa de promoción efectiva del $100 \%$, sin abandono escolar, con un bajísimo nivel de repitencia, y con una moderada tasa de sobre-edad, producto de la recepción de alumnos repetidores de otras escuelas. De los 400 alumnos aproximadamente el 75-80\% reciben AUH. Con respecto a la inasistencia escolar, cabe subrayar que señalaron que era una problemática que había estado presente en la institución, sin embargo, según personal docente, desde la escuela se habían tomado varias medidas para reducirla. Además, según uno de los directivos el mismo señaló que era una cuestión vinculada fundamentalmente a factores socioeconómicos, ya que la mayoría de los/as alumnos/as que solían faltar viven en un asentamiento precario ubicado en las cercanías de la Escuela, cuyas calles están sin asfaltar, en zona vulnerable, donde existen situaciones de necesidades básicas insatisfechas, impidiendo a los/as niños/as concurrir al establecimiento educativo cada vez que llueve. Sostuvieron que desde la escuela se realizaron y realizan todos los esfuerzos posibles para lograr que estos/as niños/as que no logran concurrir cotidianamente al establecimiento, puedan hacerlo, y en los últimos años, hubo muchísimas políticas educativas complementarias destinadas a la mejora en la inclusión y calidad escolar, sumado a ello la $\mathrm{AUH}$, que en conjunto han generado buenos resultados para reducir la inasistencia escolar comentaban en la institución.

Luego se concurrió al Cáritas (9) que desarrolla sus tareas en la Parroquia San Miguel Arcángel debido a que al mismo asisten las madres seleccionadas para obtener los servicios que éste brinda. Este Cáritas comenzó a funcionar en la mencionada Parroquia hace 35 años según datos brindados por el Sacerdote de la misma y uno de los miembros de Cáritas. Las tareas que despliega Cáritas aquí son distribución de alimentos; ropa; calzado y a su vez, información legal en situaciones donde se encuentren vulnerados derechos personalísimos de las personas que se acercan a pedir ayuda, como también acompañamiento a diferentes entes oficiales para tramitación de jubilaciones y/o pensiones, o bien trámites bancarios cuando los mismos son pedidos.

Partiendo de la información recientemente expuesta que ha sido recolectada y co-construída a partir de entrevistas como por observación etnográfica puede notarse que ambas instituciones se destacan por 
tener prácticas inclusivas que fomentan a las madres la escolarización y asistencia escolar de los/as niños/as.

\section{Acerca de las características socio-ocupacionales y educativas de los hogares}

Según las entrevistas a las 20 madres, las características constitutivas de sus hogares indicaron que todos están conformados por ambos padres y sus hijos/as. Los padres y madres habían finalizado el primario pero habían abandonado el secundario por razones económicas. En cuanto a la vivienda, todas las madres aseveraron vivir en condiciones de hacinamiento, con diversos problemas y falencias en la infraestructura del hogar. Además, señalaron que sus esposos eran trabajadores no registrados que realizaban changas y/o albañilería; mientras que 17 de ellas eran empleadas domésticas y 3 desocupadas. Con respecto a sus hijos/as, todas las madres tenían entre dos y cuatro hijos que concurrían al nivel de educación primario. Es necesario resaltar, que para que los niños y/o adolescentes obtengan el derecho para que sus madres, padres y/o tutores perciban la AUH, éstos deben encontrarse en situación de desocupación, o en la economía informal, o bien, si están en la economía formal, no pueden sobrepasar el salario mínimo vital y móvil. Por lo tanto, en estos casos estudiados todos los hogares de las madres entrevistadas cumplían con los requisitos para que las mismas sean perceptoras de la AUH.

Da cuenta de esto una de las madres:

“y si mi casa es muy humilde... dormimos todos juntos en un cuartito... mi esposo hace changas a veces o tiene que salir a trapear como yo... para mis hijos la escuela es lo más importante... Como le digo a mis hijos, tienen que terminar de estudiar para que puedan tener un buen trabajo y no estén como uno...como yo o tu papá que te manden a trapear...la educación es lo primero...tenés que tener tu titulo de secundaria y te aceptan en cualquier trabajo...y recién ahi vas a tener una casa como la gente" (Una de las madres).

De este relato puede desprenderse que las condiciones de vulnerabilidad social en la que viven están operando en las representaciones con respecto a la escolaridad de sus hijos, haciendo que le otorguen una relevancia elevada a las credenciales educativas para lograr con estas un ascenso social.

\section{Representaciones sobre la escolaridad}

\section{Acerca de las representaciones de las madres}

Cuando nos acercamos a las madres que perciben la $\mathrm{AUH}$, para realizarle las entrevistas el lugar de reunión con las mismas fue diverso, puesto que a algunas se las ubicó en la puerta de la Escuela Primaria $\mathrm{N}^{\circ} 8$ Martín Miguel de Güemes, mientras que a otras se las encontró en Caritas de la Parroquia San Miguel Arcángel, ubicada en la misma cuadra de la Escuela, lugar al que como se ha señalado con anterioridad, van todas las semanas los madres de los/as niños/as para solicitar asistencia. 
Como ya se ha indicado el total de madres relevadas a través de las entrevistas fueron 20. De entre ellas, un grupo de 14 enviaban a sus hijos/ as a la escuela desde antes de la implementación de la AUH. En estos casos no implicó una transformación, aunque sí reforzó positivamente las representaciones existentes.

Susana (10), dijo:

"la educación es algo muy importante para los nenes... para su futuro...en mi caso solo faltan a la escuela por enfermedad...la AUH es una ayuda económica, es plata que invierto en la escuela de los nenes...para los útiles, el guardapolvos...es una ayuda muy importante...con la AUH algunos empezaron a ir al colegio... igual yo antes de la asignación ya mandaba a mis hijos a la escuela..." (Una de las madres entrevistadas).

Mientras que Liliana apuntó:

"de los que conozco ya iban a la escuela antes de la asignación...es más, hasta hace poco habia mamás que ni siquiera sabian que estaba la asignación y los mandaban igual, tampoco sabian que tenían que cobrar una vez por año... yo les explico lo de la AUHy gracias a dios se llevaron una sorpresa buena, pero siempre mandaron a sus hijos con $o$ sin asignación..." (Una de las madres entrevistadas).

De tales expresiones puede inferirse que para este grupo de madres, recibir la AUH no implicó un cambio en el modo de pensar respecto de la importancia de la educación para sus hijos, porque en la práctica ya lo ejercían. Sin embargo, reforzó positivamente ese valor que le adjudicaban a la escolaridad primaria. Por otra parte, en cuanto a la dimensión económica, cobrar la Asignación significó una ayuda económica inesperada y deseada que logró resolver varios problemas de la escolaridad.

Con respecto a lo que piensan de la educación en general, da cuenta Ana de lo que expresaron todas las madres en su totalidad:

"para mi la educación es lo más importante... para progresar, para no estar parados en las esquinas fumándote, drogándote, para mi hija yo prefiero la educación, la facultad... de ahi que sea lo que ella quiera ser... eso es lo que yo quiero para mi hija... yo no terminé mis estudios y yo no quiero eso para mi hija... yo ahora trabajo, me voy a la mañana y no vuelvo hasta tarde..." (Una de las madres entrevistadas).

Todos sostuvieron que la educación formal es fundamental para tener un futuro, fue unánime la esperanza de que sus hijos continuaran sus estudios hasta un nivel universitario. Denotan que no quieren que sus hijos repitan su historia de vida. Consideran que la educación es una inversión a largo plazo que el día de mañana les posibilitará un trabajo formal y una mejor calidad de vida. Además, también señalaron que el colegio los alejaría de la calle, de la delincuencia y las drogas.

En tanto que otro grupo de madres, compuesto por 6 madres, señaló que no tenían una tradición en enviar a sus hijos a la escuela. En estos casos, las entrevistadas dijeron que lo más importante que cambió fue que a partir de la $\mathrm{AUH}$ inscribieron a los niños/as en la Escuela y que comenzaron a sostener su asistencia a la misma.

Un ejemplo de este grupo de madres es el de Claudia: 
"... para mi comenzar a cobrar la AUH fue muy importante porque me hizo llevar a mis hijas a la escuela... la verdad que fue un empujoncito... yo antes no las llevaba pero ahorita si..." (Una de las madres entrevistadas).

En cuanto a las representaciones sobre los planes sociales en general y, a la AUH en particular, se le preguntó al conjunto de 20 madres entrevistadas si con anterioridad a la aplicación de la AUH habían recibido algún plan social, como pudo ser el Plan Jefes y Jefas de Hogar, y/o el Plan familias, y las mismas respondieron que no habían recibido ninguno de ellos. No obstante tenían conocimiento de dichos planes, y señalaron que los mismos habían significado tan sólo una mínima ayuda económica. En cambio, plantearon que la Asignación era desde el punto de vista económico "una gran ayuda" que tenía beneficios adicionales que otros planes no tuvieron, en cuanto a la salud y la educación.

Liliana señaló:

"Y la AUH para mi es una ayuda para los chicos...para mi hija la escuela es muy importante, y además, sabe lo que es la AUH, porque yo le compro toda su ropa y sus cosas para la escuela con lo de la asignación... y tiene más beneficios que otros planes... más que nada en educación y en salud..." (Una de las madres entrevistadas).

\section{Acerca del Personal directivo y docente}

En cuanto a las prácticas y representaciones escolares de los hijos/ as de las madres entrevistadas, el personal directivo y docente en sus respuestas señalaron que para el caso de algunas no habría un cambio en las representaciones respecto a la escolaridad porque ya enviaban a sus hijos/as a la escuela; sin embargo, observaron un aumento en la tasa de escolaridad, lo cual implicó una mayor asistencia. Según uno de los directivos de la institución escolar, aproximadamente hubo un aumento del 35\% de la matrícula y a su vez mantenían la asistencia escolar.

Cuando se le preguntó a María qué significó la AUH para las madres, respondió:

"Para mi significa una gran ayuda económica para ellas y sus hijos..." (Una docente de la Escuela Primaria).

En lo referente a la matrícula escolar a partir de la AUH Antonia dijo:

"Luego de la AUH aumentó la matricula escolar... y los que entraron también mantuvieron la asistencia escolar... yo creo que en esos casos la AUH ha logrado modificar la visión de las madres respecto a la importancia de la educación... Para esos casos no observo grandes cambios". (Docente en función directiva de la Escuela Primaria).

Mientras que Rosa, coincidió con lo expuesto por la entrevistada anterior y señaló:

"Observamos que desde que se implementó la AUH aquellos niños a los que ibamos a buscar a sus casas comenzaron a venir por si mismos. Eso es un paso importantísimo..." (Integrante del personal del Equipo de Orientación Escolar de la Escuela). 
En síntesis, se pudo observar que las características del Barrio, la Escuela, Caritas y los hogares incidieron también junto a la percepción de la AUH en la configuración de las representaciones escolares, puesto que estas madres están inmersas en un mundo que es social, donde tanto los sistemas simbólicos, el lenguaje, el mito, etc., como también las estructuras objetivas, que son independientes de la conciencia y la voluntad de los agentes, son capaces de orientar o de coaccionar sus prácticas o sus representaciones. Es decir, estas madres tienen una génesis social que parte de los esquemas de percepción, de pensamiento y de acción que son constitutivos de lo que Bourdieu denomina habitus y de este modo configuran las representaciones escolares que las mismas producen (Bourdieu, 1988).

\section{Reflexiones finales}

Entonces, en primer lugar, se ha visualizado que en el contexto de crisis económica-política que vivió Argentina durante el 2001, los sectores con vulnerabilidad social reclamaron al Estado la implementación de planes sociales de alcance masivo que posibilitaran ingresos mínimos de subsistencia, dando origen así unos años más tarde a la AUH. En segundo lugar, se ha observado que los estudios que abordaron el impacto de la AUH en la educación soslayaron específicamente la incidencia que la misma pudo tener en las representaciones escolares del nivel primario de madres perceptoras de la AUH desde el paradigma teórico constructivista (Valles, 1997). En tercer lugar, se ha identificado que las características del Barrio, la Escuela, la Parroquia y los hogares incidieron también en la configuración de las representaciones escolares colaborando junto a la AUH a la inclusión educativa.

Ahora bien, específicamente en cuanto a la incidencia de la AUH en la escolaridad primaria en el caso de Villa Argüello, por un lado, observamos que en los casos de 14 madres de los/as niños/as que si tenían tradición de concurrir al colegio no provocó cambios en sus representaciones, sin embargo reforzó la percepción que tenían de la educación como una credencial para lograr una movilidad social ascendente.

En este sentido puede observarse que en estos 14 casos de vulnerabilidad social, la AUH ha incidido en las representaciones de las madres positivamente ya que las mismas sostienen que la $\mathrm{AUH}$ ha mejorado la inclusión educativa de sus hijos.

A nivel monetario, las 20 madres dijeron sentir una verdadera mejora económica a partir de su percepción puesto que implicó una gran ayuda para afrontar la escolaridad de sus hijos/as, concordando así con los estudios del Ministerio de Educación Presidencia de la Nación (2011), Vega et al. (2011), Fernández Blanco y Alegre (2013), Alonso y Santiago (2014) y Arroyo, (2014). Entonces en estos casos la Asignación generó un cambio económico que contribuyó a la inclusión educativa mediante el ingreso monetario, ya que el mismo se destinó para la compra de útiles escolares, fotocopias escolares, pagos de la cooperadora de la Escuela, entre 
otros, siendo una gran ayuda para la vida escolar. Por lo tanto, aquí la AUH estaría logrando su objetivo de formación de capital humano.

Por otra parte, para los casos de las 6 madres cuyos hijos/as no tenían tradición de ir a la Escuela, observamos que según las entrevistadas señalaron que lo que cambió fue que a partir de la $\mathrm{AUH}$ inscribieron a los niños/as en el colegio y comenzaron a cumplir con la asistencia escolar. En estos casos la incidencia de la AUH fue altamente positiva.

Mientras que con respecto a las representaciones sobre las políticas sociales en general y a la AUH en particular, las 20 madres sostuvieron que la misma tiene más beneficios que otros planes sociales del pasado en cuanto a la educación.

Por último, según los directivos, el equipo de orientación escolar y docentes de la escuela primaria seleccionada, también lograron advertir que la Asignación acompaño y reforzó las representaciones escolares en los casos que ya iban a la escuela, pero lo más peculiar fue que logró modificar las representaciones de las madres de los/as niños/as que no concurrían al nivel primario, acercándolos a la institución. Por lo tanto, se puedo ver que la $\mathrm{AUH}$ generó tanto continuidades como rupturas en las representaciones escolares, significando principalmente una herramienta para la inclusión social y educativa.

\section{Referencias}

Arcidiacono, P. (2017) Asignación Universal por Hijo. Rupturas y continuidades en el campo de las transferencias de ingresos en Argentina Revista Igualdad, Autonomia personal y Derechos Sociales; Lugar: Ciudad de Buenos Aires; Año: 2017 p. 25 - 45

Alonso, M. L.; Santiago, É. G. (2014) Reflexiones sobre la asignación universal por hijo desde la escuela. Entrevistas a madres de alumnos del nivel secundario Enfoques, vol. XXVI, núm. 1, pp. 111-128 Recuperado de: http://www.redalyc.org/articulo.oa?id=25933773006

Campanella, M.;Elguer, G.; Maglio, N. (2015) Políticas para una ciudadanía inclusiva. El impacto de la Ley de Educación Nacional y de la Asignación Universal por Hijo en la inclusión social en Argentina. Márgenes, 3, Recuperado de: http://www.idaes.edu.ar/margenes/N3/ Dossier_Elguer.pdf.

D’Amico, V. (2013). La política social en debate. Desigualdades, intervención estatal e inclusión social en la Argentina democrática. Cuestiones de sociologia, (9).

Deambrosi, N. (2009) 'Ni Berisso, ni La Plata: Villa Argüello': Territorio $y$ organización social en un barrio del Gran La Plata 1958- 2008 [en línea]. Trabajo final de grado. Universidad Nacional de La Plata. Facultad de Humanidades y Ciencias de la Educación. Recuperado de: http:// www.memoria.fahce.unlp.edu.ar/tesis/te.575/pdf

Féliz, M. \& López, E. (2010). ¿Por qué Neo-desarrollismo? Hacia una caracterización de la nueva modalidad de desarrollo capitalista en la Argentina reciente. VI Jornadas de Sociología de la UNLP. Universidad Nacional de La Plata. Facultad de Humanidades y Ciencias de la Educación. Departamento de Sociología, La Plata. 
Féliz, M. (2011) ¿Neo-desarrollismo: más allá del neo-liberalismo? Desarrollo y crisis capitalista en Argentina desde los 90, en THEOMAI n 23 primer semestre. Recuperado de: http://revista-theomai.unq.edu.ar/NUMERO \%2023/5_Feliz_\%2072-86.pdf

Féliz, Mariano (2012a) La dinámica del capitalismo periférico postneoliberalneodesarrollista. Contradicciones, barreras y límites de la nueva forma de desarrollo en Argentina. Herramienta (45). Recuperado de: http:// www.memoria.fahce.unlp.edu.ar/library? $\mathrm{a}=\mathrm{d} \& \mathrm{c}=\operatorname{arti} \& \mathrm{~d}=\mathrm{Jpr} 4654$

Féliz, M. (2012b), Sin clase. Neodesarrollismo y neoestructuralismo en Argentina (2002-2011) Século XXI:Revista de CiênciasSociais. vol. 2 Recuperado de: http://cascavel.cpd.ufsm.br/revistas/ojs-2.2.2/ index.php/seculoxxi/article/view/7925

Féliz, M. (2015a) Argentina neodesarrollista: Debates sobre el modelo [en línea]. La Plata: EDULP. (Libros de Cátedra. Sociales). Recuperado de: http:// www.memoria.fahce.unlp.edu.ar/libros/pm.383/pm.383.pdf

Fernández Blanco, A., \& Alegre, P. (2013). La Asignación Universal por Hijo y su impacto en el bienestar de los hogares del Partido de General Pueyrredón. Un aporte a la discusión del piso de protección social en Argentina. Centro de Documentación, Facultad de Ciencias Económicas y Sociales, Universidad Nacional de Mar del Plata.

Gasparini, L. \& Cruces, G. (2010). Las asignaciones universales por hijo en argentina: impacto, discusión y alternativas. Económica, Vol. LVI, 105-146. Recuperado de: http://economica.econo.unlp.edu.ar/ documentos/20110519025114PM_Economica_572.pdf

Gluz, N. et. al. (2014a). Avances y desafios en politicas públicas educativas: Análisis de casos en Argentina, Brasil, Colombia y Paraguay. Buenos Aires: CLACSO. Recuperado de: http://biblioteca.clacso.edu.ar/clacso/ becas/20140627050021/AvancesyDesafios.pdf

Gluz N., Rodríguez Moyano I., (2014b) Lo que la escuela no mira, la AUH "non presta”. Experiencia escolar de jóvenes en condición de vulnerabilidad social, Propuesta Educativa Número 41 - Año 23 - Jun. - Vol 1 - Págs 63 a 73 http://propuestaeducativa.flacso.org.ar/archivos/articulos/41.pdf

Harvey, D. (2014). Breve historia del neoliberalismo, Madrid: Akal.

Subsecretaría de Planeamiento Educativo (2010) Incidencia de la Asignación Universal por Hijo en la matricula escolar. Mayo. Recuperado de: http://www.slideserve.com/teagan-rich/incidencia-de-la-asignaci-nuniversal-por-hijo-en-la-matr-cula-escolar

Jodelet, Denise (1986). "La representación social: fenómenos, concepto y teoría”, en S. Moscovici, Psicología social II, Pensamiento y vida social. Psicología social y problemas sociales, Barcelona, Paidós.

Lo Vuolo, R. (2011). La asignación universal por hijo para protección social de Argentina. En: Tuñón, I. Situación de la infancia a inicios del Bicentenario: un enfoque multidimensional $y$ de derechos. Buenos Aires: Universidad Católica Argentina. Recuperado de: http://bibliotecadigital.uca.edu.ar/repositorio/ investigacion/situacion-infancia-inicios-bicentenariomultidimensional.pdf

Ministerio de Educación Presidencia de la Nación (2011). Análisis y evaluación de los aspectos educativos de la Asignación Universal por Hijo (AUH). Recuperado 
de: http://observatorio.anses.gob.ar/archivos/documentos/OBS\%20\%20000174\%20-\%20An\%C3\%A1lisis\%20y\%20evaluaci\%C3\%B3n \%20de\%20los\%20aspectos\%20educativos\%20de\%20la\%20AUH.pdf

Montes L. B., Blanco M. T., Barbuzza R., Barrozo M., Rodríguez M. \& Ugarte L. (2014) El impacto de la Asignación Universal por Hijo para la Protección Social en las instituciones escolares de Mendoza, en MILLCAYAC Revista Digital de Ciencias Sociales 1(1) p. 177-187

Mouriño, Carolina et al (2013) Pobreza Y Protección Social Universal. - la ed. - Ciudad Autónoma de Buenos Aires, CLACSO. Recuperado de: http://biblioteca.clacso.edu.ar/gsdl/cgi-bin/library.cgi? $\mathrm{e}=\mathrm{d}-11000-00---$ off-0clacso--00-1----0-10-0---0---0direct-10---4-------0-01--11-es$\mathrm{Zz}-1---20-$ about---00-3-1-00-0--4----0-0-01-00-0utfZz-8-00\&a=d\&c=clacso\&cl=CL3.4\&d=D8

Novacovsky, I., y Sobrón, C. (1999). Propuesta de un programa de transferencia directa de ingresos para la Argentina: Ingreso para el Desarrollo Humano. En Carpio, Jorge y Novacovsky, Irene, De igual a Igual. El desafio del Estado ante los nuevos problemas sociales, Sistema de Información, Monitoreo y Evaluación de Programas Sociales (SIEMPRO), Banco Mundial y Facultad Latinoamericana de Ciencias Sociales (FLACSO).

Pautassi, L, Arcidiácono P. y Straschnoy, M. (2014) Condicionando el cuidado. La Asignación Universal por Hijo para la Protección Social en Argentina. Íconos. Revista de Ciencias Sociales. vol. 2014 p. 61 - 75

Polischer, G. (2012). Estudio del impacto de la Asignación Universal por Hijo (AUH) en consumos vinculados a la Alimentación y percepción de la misma como Derecho por parte de los actores involucrados. Respuestas Estatales en torno a la Alimentación y al Cuidado. Los casos de los Programas de Transferencia Condicionada de Ingreso y el Plan de Seguridad Alimentaria en Argentina.

Rapoport M. (2010) Laspolíticas económicas de la Argentina. Una breve historia. Buenos Aires, Planeta.

Salvia, A.; Musante, B. \& Mendoza Jaramillo, A. (2013) Análisis de impacto de la AUH en materia de inseguridad alimentaria y défcit educativo. Buenos Aires: UCA. Recuperado de: http://www.uca.edu.ar/uca/common/ grupo68/files/AUH_integrado_03.pdf

Straschnoy, M. (2015). Distintas miradas, un mismo instrumento: la figura de las condicionalidades en la política social argentina. Los antecedentes de un escenario actual. Trabajo y sociedad (25), 131-148.

Terigi, F. (2009). El fracaso escolar desde la perspectiva psicoeducativa: hacia una reconceptualización situacional. Revista Iberoamericana de Educación, 50, 23-39

Tuñón, I. \& González, M. S. (2011). Efectos de las políticas de transferencias condicionadas de ingresos sobre los procesos de escolarización en las principales áreas urbanas de la Argentina (2007-2011). II Congreso de la Asociación Internacional de Sociología, Justicia Social y Democratización. Facultad. De Cs. Económicas de la UBA, Buenos Aires.

Valles, M. (1997). Técnicas cualitativas de investigación social. Reflexión metodológica y práctica profesional, Madrid.

Vega, M. C., Sícoli, M., Campisi, M. A., Colombo, S. M., Donati, M., Cein, E. G., ... \& DAntonio, M. S. (2011) Reflexión y análisis acerca de la 
implementación de políticas sociales dirigidas a la niñez y familias de la ciudad de Mar del Plata: La Asignación Universal por Hijo, alcance e impacto.

\section{Notas}

(1) Liberalización de la economía; desregulación de las finanzas; endeudamiento externo; privatizaciones; convertibilidad; disciplina en la política fiscal, entre otros. Véase Harvey, 2007.

(2) La vulnerabilidad social está compuesta por un plano estructural, que se expresa en determinadas configuraciones individuales, o de los hogares, dada por una elevada propensión a una movilidad descendente; como por un plano que se refiere a sus consecuencias en la esfera subjetiva. Es decir, la vulnerabilidad social genera sentimientos de indefensión, incertidumbre e inseguridad (Kaztman, 1999; Filgueira, 2001; González, 2009). Según el decreto 1602/09 vulnerables serían aquellos hogares cuyos jefes de hogar estén desocupados, o con trabajo informal o bien si es formal que su salario no sea superior al mínimo vital y móvil. Esta es la comunidad que pretendemos estudiar en el marco de este proyecto.

(3) Los programas de transferencia condicionada de ingresos (TIC) otorgan dinero en efectivo a grupos de población de bajos ingresos como garantia de un ingreso minimo, a cambio del cual exigen una serie de condicionantes que los beneficiarios y/o sus familias, catalogados como "pobres con hijos a cargo" deben cumplirpara recibir la ayuda, entre los que más frecuentemente se encuentran la asistencia escolar y el cuidado de la salud. Reconocen un origen comín en el Programa de Educación, Salud y Alimentación (Progresa), que en el año 1997 se instaló a nivel nacional en México y actualmente está reconvertido en el llamado Programa Oportunidades, tal como afirman Minujin, Alberto; Davidziuk, Alejandra y Delamónica, Enrique, "El Boom de las Transferencias de Dinero Sujetas a Condiciones. ¿De qué Manera Estos Programas Sociales Benefician a los Niños y Niñas Latinoamericanos?". Salud colectiva [online]. vol.3, n.2 [citado 2011-06-23], 2007, pp. 121-131 y Lo Vuolo, Daniel Raventosy Pablo Yáñez, "El Ingreso Ciudadano-Renta Básica ante la crisis económica y los ataques a los derechos sociales y laborales", disponible en www.sinpermiso.info, 12 de septiembre 2010. Para más véase D’ Amico (2014). Para más sobre Política de Transferencia Condicionada de Ingresos véase Pautassi, Aracidiácono y Straschnoy (2014) y Arcidiácono (2017).

(4) En su momento era de \$180 para niños menores de 18 años, y de un monto especial para chicos discapacitados sin límite de edad, pero fue ajustándose al compás de la inflación. Mientras que en 2013 el monto alcanzó a \$460. Cabe señalar que en julio de 2013 el costo de la canasta básica era de \$1.679,36 (INDEC).

(5)se conservará el anonimato por acuerdo con los sujetos.

(6) Durante el período 2013-2014 se llevó adelante la presente investigación, la cual formó parte de un proyecto mayor en el marco de la elaboración de la tesina para la obtención del título de grado de la autora de éste trabajo.

(7)Se desconocen las razones por las cuales se lo denominaba de ese modo.

(8)Para Féliz \& López (2010) varios años de crecimiento acelerado y sostenido marcaron el inicio de una nueva modalidad de desarrollo capitalista periférico en Argentina denominada Neodesarrollista (2002-2009). Ese modelo se sostuvo sobre la base de un tipo de cambio real alto, el superávit fiscal sostenido y la renegociación para el pago de la deuda pública externa. Dicha conceptualización se relaciona más con los cambios en la forma concreta del Estado que con las modificaciones en la estructura y dinámica del capital, que parecen evidenciar más continuidades que rupturas en relación a la fase neoliberal de los años noventa. Con respecto a las políticas macroeconómicas del período Neodesarrollista reconocen el tipo de cambio elevado, el superávit fiscal y el esquema 
de subsidios y la política de (des)endeudamiento. En relación a las políticas sociales, la implementación del Plan Jefes y Jefas de Hogar Desocupados, el Plan Familias, el Programa Argentina Trabaja y la Asignación Universal por Hijo. En cuanto a políticas laborales, destacan la reapertura de las paritarias, la renegociación de los convenios colectivos y los incrementos del salario mínimo, entre otras. Es más claro aquí que el accionar estatal permite la estabilización -conflictiva- del patrón de acumulación, sintetizando las posiciones de los trabajadores formales y las clases dominantes.

(9) Cáritas Argentina es el organismo oficial de la Iglesia Católica que lleva adelante la pastoral caritativa. Cuenta con más de 32.000 voluntarios que están presentes en las 66 diócesis de la Iglesia Argentina y canaliza su acción a través de más de 3.500 parroquias, capillas y centros misionales que permiten el trabajo directo con las familias y personas que viven distintas situaciones de pobreza o exclusión en todo el país. Cáritas lleva adelante proyectos e iniciativas de promoción humana acompañando microemprendimientos productivos y de autoconsumo, brindando capacitación laboral, formación en ciudadanía, talleres de alfabetización para adultos, becas escolares y universitarias, espacios educativos y apoyo escolar, cuidados preventivos en salud y alimentación y asistiendo con ayuda inmediata ante situaciones de extrema pobreza o emergencias climáticas (CÁRITAS ARGENTINA).

(10) Los nombres de los entrevistados fueron modificados para preservar su identidad. 